\title{
Figement syntaxique, sémantique et pragmatique
}

Gabriela Soare et Jacques Moeschler

\section{(2) OpenEdition \\ Journals}

Édition électronique

URL : http://journals.openedition.org/pratiques/2808

DOI : $10.4000 /$ pratiques. 2808

ISSN : 2425-2042

\section{Éditeur}

Centre de recherche sur les médiations (CREM)

\section{Édition imprimée}

Date de publication : 15 décembre 2013

Pagination : 23-41

Référence électronique

Gabriela Soare et Jacques Moeschler, «Figement syntaxique, sémantique et pragmatique », Pratiques [En ligne], 159-160 | 2013, mis en ligne le 30 juin 2016, consulté le 19 avril 2019. URL : http:// journals.openedition.org/pratiques/2808; DOI : 10.4000/pratiques.2808 


\section{Figement syntaxique, sémantique et pragmatique}

\section{Gabriela Soare Jacques Moeschler}

Département de linguistique Université de Genève

\section{Introduction}

Des exemples tels que ceux en (1)-(6) sont communément décrits comme ayant un sens idiomatique, tant en linguistique traditionnelle qu'en linguistique générative :

(1) Le tribunal a rendu justice aux épargnants.

(2) Le général a donné ordre aux soldats d'attaquer.

(3) Jean a cassé sa pipe.

(4) Jean a mis les pieds dans le plat lors de la réunion.

(5) Tel est pris qui croyait prendre.

(6) La poudre a parlé.

Du point de vue sémantique, ces exemples se comportent différemment, des plus grammaticalisés, comme en (1) et (2), aux plus fortement figés, comme en (3) et (4), et jusqu'à des cas de proverbes, comme en (5) et (6). Si (1) et (2) sont caractérisés par une sémantique compositionnelle, d'où souvent l'appellation de locutions verbales (par exemple, Gross 1996, Gaatone 1981, 2000, Moeschler 1992a, parmi d'autres), dans les autres cas, leur sens n'est pas facilement déductible du sens littéral de leurs composantes par les règles usuelles de la sémantique compositionnelle. Ce comportement peut être dû à plusieurs facteurs : (a) les éléments qui font partie de l'expression idiomatique (ou idiome) ont plusieurs sens (parfois non-spécifiés), dont un seul est compatible avec le contexte ; (b) l'idée la plus généralement acceptable est que les éléments qui composent l'expression idiomatique ont des sens littéraux, mais que ceux-ci ne sont pas impliqués dans l'interprétation de l'expression idiomatique. Ce type d'expression idiomatique est appelé une métaphore morte ou métaphore figée.

Les locutions verbales et les expressions idiomatiques existent dans toutes les langues naturelles et dans tout style de langue. Le problème qu'elles posent n'est pas que le sens ne peut pas être inféré sur la base de mécanismes habituels, mais qu'à la différences des autres items lexicaux, la plupart des idiomes et des locu- 
tions verbales ont une structure interne qui interagit avec des mécanismes syntaxiques et sémantiques de la langue en question.

Cette contribution vise à montrer quelques problèmes que les locutions verbales et les expressions idiomatiques posent du point de vue linguistique. Le but est de remettre en question certaines idées reçues sur leur fonctionnement syntaxique et sémantique, et de considérer les conséquences de telles observations sous l'angle de la relation entre la linguistique et la pragmatique (les inférences). Plus précisément, l'argument que nous développerons est que la tendance au figement semble mettre en jeu un processus de restriction pragmatique d'un ensemble d'implicatures faibles menant à un développement nouveau et conventionnel de la forme propositionnelle de l'énoncé, à savoir son explicature de base.

Dans la section 2, on examinera quelques propriétés du figement en essayant de comprendre comment elles interagissent et quel rôle elles jouent pour la compréhension des phénomènes linguistiques : selon la syntaxe (Chomsky 1980, Ruwet 1983, parmi d'autres) et la sémantique (non-compositionnelle). Dans la section 3 , on reviendra sur les propriétés sémantiques des expressions idiomatiques, notamment leur non-compositionnalité, l'absence d'autonomie référentielle et le rapport entre leur sens littéral et non-littéral. La section 4 examinera les traitements pragmatiques classiques des idiomes, comme convention de langue ( $v s$ convention d'usage), comme nouveau sens littéral, et finalement comme implicature conversationnelle particulière. Nous terminerons dans la section 5 par discuter d'une approche nouvelle des expressions figées, qui conduit, via la notion de concept $a d$ $h o c$, à les considérer comme une contribution au sens explicite de l'énoncé : leur sens serait une explicature de base, à savoir un enrichissement de la forme propositionnelle de l'énoncé ${ }^{(1)}$.

\section{Propriétés syntaxiques et sémantiques}

Tout au long de la discussion, on adoptera la distinction de Ruwet (1983) entre expressions idiomatiques sémantiques (ExiMs, ou M signifie «meaning »), telles que foutre le camp, mettre les pieds dans le plat, manger des pissenlits par la racine, etc. et expressions idiomatiques syntaxiques (ExiFs, ou F signifie « form»), telles que rendre justice, prêter assistance, etc. Les ExiFs correspondent à ce qu'on a appelé dans 1'Introduction locutions verbales. Dorénavant, on utilisera par abréviation les termes d'Exi (pour " expression idiomatique »), et respectivement de Exim et de ExiF. Généralement, le sens des composantes d'une ExiM ne permet pas d'interpréter leur combinaison. Une ExiF est une expression qui présente l'une ou l'autre idiosyncrasie syntaxique, qu'elle soit opaque ou non du point de vue sémantique. Plus exactement, une propriété syntaxique assez saillante des ExiFs est représentée par l'absence de déterminant dans le NP objet, cf. rendre justice, rendre hommage, prêter assistance, donner tort, faire honneur, etc. Sémantiquement, un bon nombre de ces ExiFs ne sont pas opaques. Certaines expressions sont à la fois des ExiFs et, à divers degrés, des ExiMs, telles que chercher noise ${ }^{(2)}$, prendre ombrage.

(1) Une explicature est un enrichissement pragmatique, donnant lieu à la forme propositionnelle de la phrase énoncée. Elle correspond aux hypothèses communiquées explicitement. Les implicatures rassemblent au contraire toutes les hypothèses communiquées implicitement (prémisses implicitées et conclusions implicitées).

(2) L'usage de chercher noise est plus rare et sémantiquement moins transparent, pour des raisons liées au changement linguistique (noise n'est plus utilisé en dehors de l'ExiF/M cher- 
Plusieurs théories ont été proposées pour rendre compte du comportement des expressions idiomatiques. Par exemple, les approches fondées principalement sur la théorie de Chomsky (1980) considèrent que la nature idiosyncratique des Exis est une propriété lexicale et que cette idiosyncrasie devrait être formulée une seule fois, à un seul niveau, la structure-D (structure profonde). Par conséquent, les différentes formes que peut prendre une expression idiomatique devraient se référer à ce niveau, dit canonique, et c'est la théorie syntaxique qui fournit les moyens de relier les différentes formes de l'idiome à sa forme canonique (pour des arguments contre la théorie de Chomsky, voir, par exemple, Ruwet 1983 ; 1'approche de Gazdar, Klein, Pullum and Sag 1985, Pulman 1993 formulée dans les termes de la Grammaire syntagmatique généralisée ; l'approche de la Grammaire lexico-fonctionnelle de Bresnan 1982, etc.). Vu que plusieurs théories se basent sur l'approche de Chomsky (1980), c'est cette approche qui sera considérée plus en détail dans la discussion.

Selon Chomsky (1980), c'est au niveau de la structure-D (structure profonde) que les expressions idiomatiques sont non « disséminées » et que des règles lexicales opèrent sur la structure- $\mathrm{D}^{(3)}$. Ces règles opèrent sur des constituants et non pas sur des parties arbitraires de l'arbre syntaxique. En d'autres termes, les expressions idiomatiques sont des unités sémantiques. Selon cette théorie, il y a une seule règle par Exi.

Examinons maintenant les Exis relativement à deux transformations syntaxiques, utilisées régulièrement (en plus de la pronominalisation par exemple) comme test de leur caractère non-disséminé : le passif et le clivage.

Considérons premièrement le comportement des Exis vis-à-vis du passif. En règle générale, ce sont les Exis moins figées qui peuvent subir la passivation, tandis que les Exis figées n'admettent pas le passif. A l'intérieur des ExiFs (ou locutions verbales), une distinction doit être faite entre les Exis qui permettent cette opération et celle qui soit ne la permettent pas soit la permettent dans certaines conditions. Un groupe d'ExiFs de forme V Det NP admettent naturellement la passivation. Des Exis du type faire la lumière, donner le coup de grâce sont très facilement passivables, comme montré en $(7)^{(4)}$.

(7) a. La lumière n'a pas été faite sur les écoutes téléphoniques illégales.

b. Le coup de grâce a été donné aux journalistes.

Le deuxième groupe d'ExiFs est représenté par les expressions où l'objet est possible sans déterminant, comme dans prendre part, prendre contact, rendre justice, rendre hommage, faire justice, etc. ${ }^{(5)}$ Dans ce cas, la passivation ne peut s'effectuer que si le déterminant apparaît. La présence du déterminant au passif ne semble pas entraîner d'effets d'interprétation. Une autre condition syntaxique qui facilite la passivation est la modification, qui entraîne, dans les deux cas — dans la phrase passive, ainsi que dans la phrase active - la présence d'un déterminant :

(8) a. Les Russes ont pris part/une part importante aux discussions de paix.

cher noise). Qui sait d'ailleurs que noise signifie anciennement le bruit causé par une querelle, un tumulte (TLS) ?

(3) Le terme « expression non disséminée » signifie expression formée de constituants contigus.

(4) Une expression du type V Det DP comme donner le coup de grâce admet l'opération de passivisation à cause de la présence du déterminant qui est intimement lié à la modification du $\mathrm{N}$ coup .

(5) Voir cependant les exemples de phrases passives en (10). 
b. *Part a été prise par les Russes aux discussions de paix.

c. Une part importante a été prise par les Russes aux discussions de paix.

En partant de l'idée de base selon laquelle il est souvent possible d'établir une correspondance de terme à terme entre les constituants de l'Exi et les éléments de son sens idiomatique, on observe que le passif est admis lorsque les constituants de l'Exi jouissent d'une relative autonomie référentielle. C'est aussi ce manque d'autonomie référentielle des constituants qui est la clé du degré de figement des Exis. En d'autres termes, le degré de figement peut influer sur la possibilité de passivation d'une Exi. Ceci est une condition nécessaire, mais non suffisante. Pour illustration, à la différence de $(9 a),(9 b)$ et $(9 c)$, le NP sujet en (9d) jouit d'une certaine autonomie référentielle. Il faudrait noter que même si mettre les voiles est une Exi plus transparente que foutre le camp, la passivation n'est admise dans aucune des deux.

(9) a. * Le camp a été foutu par Jean.

b. * Les voiles ont été mises par Jean.

c. * Sa pipe a été cassée par Jean.

d. La glace a été brisée par Jean.

Pour revenir au cas de l'absence du déterminant en (8b) et à l'impossibilité de passivation, celle-ci peut être attribuée au sens du verbe prendre dans prendre part, qui a un sens lexicalisé et non pas son sens littéral ou plein ("saisir»ou « s'emparer »). Comme on l'a vu, si d'autres conditions syntaxiques (ou sémantiques) sont remplies, la passivation est admise, en l'occurrence la modification du $\mathrm{N}$ part qui entraîne la présence obligatoire du déterminant en $(8 \mathrm{c})$.

Pourtant, dans certaines ExiFs sans déterminant, le passif est accepté. Ceci est montré en (10a) et (10b). (10c) et (10d) représentent des phrases absolues, qui sont, elles aussi, acceptables.

(10) a. Justice a été faite par la cour.

b. Justice a été rendue par la cour.

c. On croyait tous justice faite.

d. On croyait tous justice rendue.

L'explication de leur acceptabilité tient, d'après Ruwet (1983), au type de passif, plus exactement au fait qu'il y a un adjectif statif en (10) ${ }^{(6)}$.

(6) Il faut souligner l'existence d'expressions idiomatiques, et surtout d'ExiFs relativement transparentes, où ni le passif, ni la phrase active ne sont acceptés, mais où la construction absolue produit un résultat acceptable. Ceci est montré en (i) et (ii) ci-dessous :

(i) a. Tout compte fait, je démissionnerai bientôt.

b. * Tout compte a été fait (sur ma démission).

c. * J'ai fait tout compte (sur ma démission).

(ii) a. Toute honte bue, Jean a quitté la sale.

b. * Toute honte a été bue (par Jean).

c. * Jean a bu toute honte.

Ce genre de cas est discuté par Ruwet (1983), qui explique l'acceptabilité des phrases en (a) par le fait que dans tous ces cas il y a un prédicat statif. La construction absolue joue un rôle amélioratif même pour certaines ExiMs. Dans l'exemple ci-dessous, le passif n'est pas permis en (iiib), mais dans la construction absolue en (iiia), la phrase est acceptable ou presque :

(iii) a. (?) Une fois la croûte cassée, ils se sont mis à manger.

b. ?* La croûte a été cassée par le père et ils se sont mis à manger.

c. Le père a cassée la croûte et ils se sont mis à manger. 
Un autre facteur qui est lié sémantiquement au facteur d'autonomie référentielle, qui intervient dans l'(im)possibilité de passivation, concerne le type d'objet. Si, comme en (9c), l'objet désigne une propriété inaliénable du réfèrent du sujet, le passif n'est pas admis (Keenan 1975, Doetjes et Rooryck 2003, parmi d'autres). (11)-(13) sont des cas supplémentaires pour illustrer cette idée :

(11) a. Jean a levé le bras droit.

b. * Le bras droit a été levé par Jean.

(12) a. Jean a donné sa langue au chat.

b. * Sa langue a été donnée au chat par Jean.

(13) a. Jean a mis les pouces.

b. * Les pouces ont été mis par Jean.

Un autre cas de construction où le type d'objet entre en jeu concerne des cas comme (14) et (15).

(14) a. Ce dîner m'a coûté les yeux de la tête.

b. * Les yeux de la tête m'ont été coûtés par ce dîner.

(15) a. Ce dîner m'a coûté une fortune.

b. * Une fortune m'a été coûtée par ce dîner.

L'impossibilité de la passivation dans ces cas est due au fait que le NP objet est caractérisé par le trait [+quantité/+mesure] et, par conséquent, a le comportement d'un quasi-adverbial.

De plus, un NP indéfini d'une phrase active n'est, en règle générale, pas passivable. Or, les Exis faire un tabac/un bide contenant un objet indéfini peuvent être passivées une fois qu'elles sont soumises à des contraintes pragmatiques bien particulières. Ainsi, ces Exis deviennent acceptables dans une phrase relative réduite au passif, comme dans Mon impresario ne s'est pas remis du bide fait par mon film (exemple pris du Ruwet 1983).

Pour conclure, le choix et l'interprétation du déterminant dans le NP sujet d'une phrase passive échappent aux contraintes purement syntaxiques et mettent en jeu des conditions pragmatiques, comme on le verra dans la section 4 .

Une autre propriété syntaxique des expressions idiomatiques concerne leur comportement dans les phrases clivées. Étant donné que les expressions idiomatiques ont une distribution unique et sont engendrées au niveau de la structure-D, selon la théorie de Chomsky, l'opération de clivage ne devrait pas être permise. Or, les exemples en (16) montrent que la focalisation du syntagme nominal (NP) monts et merveilles de 1'Exi promettre monts et merveilles et du syntagme prépositionnel dans le ruisseau de l'Exi trouver quelqu'un dans le ruisseau produisent de phrases relativement acceptables.

(16) a. ?(?) C'est monts et merveilles que nous a promis la direction. ${ }^{(7)}$

b. C'est dans le ruisseau que Pierre a trouvé Lucie.

Pourtant toutes les Exis ne permettent pas la focalisation. Ceci est montré en (17)-(19) :

(17) ?? C'est hommage que l'armée a rendu aux soldats disparus.

(7) La presence d'un déterminant rend cette phrase naturelle :

(i) C'est des monts et des merveilles que nous a promis le président. 
(18) a. *? C'est le taureau qu'il a pris par les cornes.

b. *? C'est les pieds qu'il a mis dans les plats.

(19) a. ?? C'est par les cornes qu'il a pris le taureau.

b. ?? C'est dans les plats qu'il a mis les pieds. [Ruwet (1983, exemples (21b, 21c))]

L'opération syntaxique de déplacement du syntagme nominal ou prépositionnel en (18-19) ne donne pas un résultat grammatical. Ce qui semble être en jeu ici est la nature sémantique de l'élément clivé. Il présuppose l'existence d'un ensemble d'éléments sur la base desquels il est identifié et contrasté. Or, ces exemples suggèrent l'absence d'un tel ensemble d'éléments. En (16a), le verbe promettre a son sens habituel, et le NP coordonné monts et merveilles s'oppose implicitement à d'autres éléments, tels que (promettre) des bons résultats, (promettre) une bonne année. En (16b), le verbe trouver a son sens habituel, ce qui permet au PP d'être facilement opposable à d'autres éléments, trouver quelqu'un à la cafétéria/dans les bois, etc.

La modification d'une partie de l'Exi peut engendrer un résultat grammatical. Par exemple, si le DP en (17a) est modifié, la phrase devient une phrase plus acceptable :

(20) (?) C'est par les cornes de la syntaxe qu'il a pris le taureau de la sémantique.

En (21), le NP contenant un déterminant est modifié par l'adjectif retentissant et la phrase devient acceptable.

(21) C'est un hommage retentissant que l'armée a rendu aux soldats disparus.

La modification d'une partie de l'Exi a pour effet de rendre l'élément en question sémantiquement plus lourd et, par conséquent, susceptible d'être plus facilement contrasté avec d'autres éléments. Le syntagme focalisé des clivées doit donc bénéficier d'une certaine autonomie référentielle.

Un autre aspect important de la syntaxe des Exis concerne leur ordre d'engendrement syntaxique. Il y a des expressions idiomatiques qui présentent l'ordre obligatoire PP > NP, comme exemplifié ci-dessous :

(22) a. Jean a fait d'une pierre deux coups.

b. * Jean a fait deux coups d'une pierre.

(23) a. Jean fait de nécessité vertu.

b. * Jean fait vertu de nécessité.

(24) a. Marie fait contre mauvaise fortune bon cœur.

b. ${ }^{*}$ Marie fait bon cœur contre mauvaise fortune.

Les phrases (22a), (23a) et (24a) ont un sens idiomatique, dont atteste l'impossibilité de permutation syntaxique des deux arguments, même dans une lecture littérale. D'autres expressions idiomatiques présentent l'ordre inverse, NP $>$ PP, comme illustré en (25)-(29) :

(25) a. Jean a fait flèche de tout bois.

b. * Jean a fait de tout bois flèche.

(26) a. Jean a pris le taureau par les cornes.

b. * Jean a pris par les cornes le taureau.

(27) a. Jean cherche midi à quatorze heures.

b. * Jean cherche à quatorze heures midi.

(28) a. Jean jette de la poudre aux yeux.

b. * Jean jette aux yeux de la poudre. 
(29) a. Jean a mis les pieds dans les plats.

b. * Jean a mis dans les plats les pieds.

L'ordre NP > PP est obligatoire même dans les Exis où le NP est libre, comme mettre XP en pièces/dans le coup/sur le carreau. Ceci est illustré en (30) et (31):

(30) a. Jean a mis un homme en pièces.

b. * Jean a mis en pièces un homme.

(31) a. Jean a mis sa petite amie dans le coup.

b. * Jean a mis dans le coup sa petite amie.

Le fait qu'un seul ordre soit possible, soit V NP PP, soit V PP NP, ne peut pas être dû à la syntaxe, mais on peut attribuer ce facteur à la pragmatique ${ }^{(8)}$. Du moins en (22)-(24), où l'on peut parler de rapport d'antériorité. Par exemple, en (22) et (23), la relation entre une pierre et deux coups, d'une part, et entre la nécessité et la vertu, d'autre part, est une relation de cause à effet. En (24), la relation en jeu est celle d'opposition. De la même façon, les Exis en (25)-(31) sont construites sur certains types de rapport : en (25), il y a une relation de résultat-matière, en (26) il $\mathrm{y}$ a une relation d'objet (ou thème) - partie de 1'objet et en (27)-(31) une relation de thème (ou patient) - location.

Pour conclure, les particularités syntaxiques et sémantiques des Exis sont des conditions nécessaires, mais non suffisantes : les conditions pragmatiques de leur emploi doivent donc être prises en compte.

Nous avons analysé quelques propriétés syntaxiques et sémantiques des ExiFs et des ExiMs. Dans les paragraphes suivants, on verra que les Exis sont, à divers degrés, analysables, et que donc le sens littéral de leurs parties reste dans bien des cas pertinent. En d'autres termes, les expressions idiomatiques se prêtent à l'analyse syntaxique et sémantique, ainsi qu'à l'analyse pragmatique, sur la base de leur structure interne, du sens littéral de leurs constituants et des types de rapports qui entrent en jeu (par exemple, des rapports métaphoriques, métonymiques, etc.) entre le sens littéral et le sens idiomatique ou figé. Concernant ce dernier aspect, celui du sens figé, on verra également que celui-ci est le résultat d'un processus de restriction pragmatique d'un ensemble d'implicatures faibles menant à un développement nouveau et conventionnel de la forme propositionnelle, à savoir de son explicature de base.

\section{La sémantique des expressions idiomatiques}

Les Exis ont toujours été considérées du point de vue sémantique comme noncompositionnelles. Par exemple, Ruwet $(1983,34)$ considère qu'étant non-compositionnelles, elles doivent être apprises les unes après les autres par cœur :

«Les expressions idiomatiques doivent être plus ou moins apprises une à une, par cœur ; le sujet parlant apprenant une langue doit y être exposé, découvrir qu'elle existe dans la langue sous telle forme plutôt que sous telle autre. »

La première exigence est donc de tester leur caractère compositionnel ou non compositionnel.

(8) L'existence d'un ordre possible avec certaines expressions et d'un autre ordre avec d'autres expressions soulève la question de leur engendrement syntaxique et constitue pour Ruwet un argument contre la thèse de Chomsky selon laquelle les expressions idiomatiques ne sont pas disséminées en structure-D. 
Cette propriété sémantique de non-compositionnalité est liée à une seconde propriété sémantique, que possèdent les arguments internes des expressions libres, non idiomatiques : leur autonomie référentielle (Milner 1982).

Enfin, la troisième propriété sémantique, si l'on veut tester le caractère non compositionnel et non autonome de leur argument interne, est la possibilité de leur usage littéral.

Examinons chacune de ces propriétés. Nous essayerons de montrer que si la contrainte d'autonomie référentielle est la plus forte, la non-compositionnalité n'est pas absolue (il y a pour nous un continuum entre Exis complètement noncompositionnelles et Exis plus ou moins compositionnelles, celles notamment qui acceptent une interprétation littérale). Quant à l'analyse de l'usage littéral, nous la poursuivrons dans la dernière section, à propos de la pragmatique des Exis.

Un bon test de non-compositionnalité des Exis est l'impossibilité de leur traduction littérale d'une langue à l'autre. Jean-Loup Chiflet (1985) a fait un recensement très drôle de telles impossibilités, pour une traduction du français à l'anglais :

(32) a. passer du coq à l'âne

b. to pass from cock to donkey

c. to change the subject

(33) a. faire un bide

b. to make a stomach

c. to have egg on your face

Cela dit, si les Exis se comportent sémantiquement de manière non compositionnelle, la question est de savoir comment elles sont porteuses d'un sens. Nous verrons précisément comment le passage du sens littéral au sens figuré peut s'envisager d'un point de vue pragmatique, mais du point de vue de la sémantique, il n'y a que deux solutions possibles. La première passe par une attribution globale de sens à l'ensemble de la construction, qui fonctionnerait comme un prédicat complexe, dont le sens serait aussi arbitraire que celui attribué à une expression simple. La seconde consiste à associer à l'expression un contenu (concept) dont le déclencheur ne serait pas le prédicat, mais l'argument ${ }^{(9)}$.

La première solution reçoit des objections évidentes, qui sont liées à l'association d'un sens arbitraire à une expression figée. Même si, pour la plupart de ces expressions, leur origine ou statu nascendi n'est pas clair, car mal documenté, il est assez surprenant de considérer qu'il n'y a aucune relation entre le sens compositionnel et leur sens idiomatique. Prenons le cas de casser sa pipe. Les tests sur son caractère idiomatique sont clairs : la passivation donne des résultats bizarres (pour des raisons liées à la violation de la règle de c-commande), la pronominalisation, la dislocation à droite ou à gauche et le clivage forcent l'interprétation littérale, et la substitution du prédicat et de son argument interne par un synonyme rend à l'expression un sens non idiomatique :

(34) a. * Sa pipe a été cassée par Max.

b. \# Il l'a cassée, sa pipe. ${ }^{(10)}$

c. \# Sa pipe, il l'a cassée.

d. \# C'est sa pipe qu'il a cassée.

(9) Cf. Moeschler (1992b) pour une telle approche, que nous ne développerons pas ici Nous reviendrons en section 5 sur la première option.

(10) Le signe \# est utilisé pour indiquer le changement de sens, ici le seul accès au sens littéral. 
(35) a. Max a cassé son brûle-gueule.

b. Max a brisé sa pipe.

Le dernier résultat (substitution de synonymes impossible) est assez paradoxal, car on pourrait justement arguer du fait que la question de l'interprétation des Exis n'est pas une question de signification mais de forme. La conjonction de formes particulières, dans un environnement particulier, est nécessaire à son association à un sens spécifique.

Mais regardons maintenant de plus près une interprétation sémantique possible. La comparaison de casser sa pipe et de son équivalent anglais to kick the bucket (littéralement «jeter un coup de pied dans le seau ») peut être éclairante. Supposons les scénarios possibles, qui ne sont que des reconstructions sans fondements diachroniques sur l'origine même de l'expression. Ces deux expressions décrivent des achèvements, et comme tout achèvement, elles impliquent un changement d'état, ou un résultat.

Prenons maintenant un équivalent littéral de ces expressions :

(36) Max a cassé ses skis.

(37) Bill kicked the ball.

Le résultat, dans les deux cas, est que les skis sont cassés et que le ballon a subi une trajectoire impliquant un déplacement d'un point à un autre. Contextualisons maintenant les deux expressions idiomatiques, avec leur signification idiomatique MOURIR SUBITEMENT. Si nous pouvons envisager la mort comme le résultat d'un achèvement, on peut alors se représenter l'événement de casser sa pipe comme un indice d'un changement d'état. Le sens figuré serait donc le résultat d'un processus, et plus précisément le résultat du changement d'état d'un achèvement. D'un autre côté, to kick the bucket est l'événement cause du résultat qu'est la mort (on peut imaginer une représentation stéréotypique où un suicidaire est sur un seau, corde enroulée au cou, et donne un coup de pied au seau pour se pendre).

Cette description n'a aucun fondement diachronique et la question n'est pas la reconstruction du sens de ces expressions. La question est de savoir s'il existe une motivation sémantique entre le sens descriptif de l'expression et son sens métaphorique. D'un point de vue sémantique, nous répondons positivement : l'expression décrit soit un événement qui est la cause, soit un événement qui est l'indice d'un résultat correspondant au sens de l'expression idiomatique.

Y a-t-il maintenant une corrélation entre la non-compositionnalité sémantique, reformulée en termes de relations sémantiques entre le sens littéral et le sens figuré, et la non-autonomie référentielle? Nous avons examiné les conséquences syntaxiques de cette propriété sur les computations syntaxiques que ne permettent pas les Exis, et notamment le passif. Nous aimerions maintenant en montrer les conséquences sémantiques.

L'autonomie référentielle est une propriété sémantique fondamentale permettant notamment d'expliquer le comportement des noms ordinaires, par opposition aux noms de qualité (Keenan E. 1975, Milner 1982, Moeschler \& Reboul 1994, chapitre 14). Le contraste entre ces deux types de nom est donné en (38) et (39):

(38) Jean a planté la tente.

(39) Jean est un idiot.

En (38), la tente est une expression référentielle autonome : elle réfère à une en- 
tité particulière, la tente de Jean. Il est possible d'y faire référence par un pronom anaphorique, comme en (40) :

(40) Jean a planté la tente . $_{\mathrm{i}}$ Elle $_{\mathrm{i}}$ se monte en 5 minutes.

En revanche, un idiot ne peut pas être 1'antécédent d'un pronom : en (41), il réfère à Jean, car un idiot est un nom de qualité, non une expression référentielle, et ne peut donc pas être l'antécédent du pronom :

(41) $\mathrm{Jean}_{\mathrm{i}}$ est un idiot $\mathrm{j}_{\mathrm{j}} \mathrm{Il}_{\mathrm{i} / *_{\mathrm{j}}}$ a quitté Marie.

La question est maintenant de savoir si cette propriété sémantique est maintenue dans les Exis. Selon le test de la reprise anaphorique, si l'argument interne d'une Exi est autonome référentiellement, on doit pouvoir en faire la reprise anaphorique dans une autre phrase. Or les données montrent clairement que ce n'est pas le cas:

(42) a. Max a cassé sa pipe. Elle était en onyx.

b. ?? Max a cassé sa pipe. C'était une chienne de vie.

(43) a. Max a cassé la glace. Elle était très épaisse.

b. ?? Max a cassé la glace. Elle rendait tout le monde timide et malheureux.

(44) a. Max a pris le taureau par les cornes. Elles étaient très pointues et il s'est blessé.

b. ?? Max a pris le taureau par les cornes. Elles étaient insolubles, mais il trouva la solution en travaillant d'arrache-pied.

Les pronoms dans les exemples (a) donnent tous lieu à une interprétation littérale, non figurée. L'interprétation idiomatique est donc suspendue, et c'est l'interprétation littérale qui émerge. Dans les exemples (b), le pronom reprend formellement les propriétés morphologiques de 1'argument interne (pipe, glace, cornes). Même s'il réfère à une entité en relation (pragmatique) avec l'argument interne, le pronom ne peut assurer la cohérence du discours ${ }^{(11)}$.

L'absence d'interprétation référentielle de l'argument interne montre bien que l'interprétation figurée viole un principe fondamental de la détermination de la signification des phrases. L'interprétation descriptive, vériconditionnelle, suppose non seulement l'interprétation référentielle des arguments, mais aussi la satisfaction de la sémantique du prédicat: Max a planté la tente est vrai si et seulement si la tente appartient à l'ensemble des entités qui sont plantées, et la phrase est vraie si Max appartient à l'ensemble des entités qui sont vraies du prédicat planter la tente.

Dans aucune des Exis, même les plus proches des expressions libres, comme casser la glace, ni le principe de compositionnalité, ni l'autonomie référentielle ne sont satisfaits. Le dernier critère à tester porte sur la possibilité d'une interprétation littérale.

Le test de l'interprétation littérale permet en fait de discriminer différents types d'Exis. Prenons la différence entre briser la glace et promettre monts et merveilles. La première peut être interprétée littéralement, comme en (45):

(11) En (42b), le démonstratif ce est l'anaphore de VIE, connecté sémantiquement au résultat MORT. En (43b), le pronom elle est censé référer à ATMOSPHERE, AMBIANCE. Enfin, en (44b), elles renvoie à PROBLEMES, impliqué par le sens idiomatique. Mais dans une interprétation pragmatique via le principe d'identification (Fauconnier 1984), où le pronom $i l s$ s'accorde non avec le déclencheur, mais avec la cible (PROBLEMES), la suite est tout aussi inacceptable (i) :

(i) ?? Max a pris le taureau par les cornes. Ils étaient insolubles, mais il trouva la solution en travaillant d'arrache-pied 
(45) a. Marie a brisé la glace en servant une coupe de champagne.

b. Marie a brisé la glace avec une masse.

Les ajouts (en servant une coupe de champagne, avec une masse) désambiguïsent l'interprétation figurée (45a) et littérale en (45b). En revanche, (46) ne peut recevoir d'interprétation littérale :

(46) a. Max promis monts et merveilles à sa fiancée : une grande maison, plein d'enfants, et beaucoup d'argent.

b. Max a promis monts et merveilles à sa fiancée : le Mont-Blanc, le Cervin, la caverne d'Ali Baba, la bibliothèque d'Alexandrie.

Si (46a) ne pose pas de problèmes, ce n'est pas le cas de (46b), qui ne s'interprète que figurativement et non littéralement. Même dans cette situation, il faudrait une interprétation via la coercion sémantique, mais l'interprétation est vraiment peu claire dans ce sauvetage extrême de l'interprétation littérale :

(47) a. Max a promis à sa fiancée d'escalader/ de la déposer en hélicoptère sur le MontBlanc.

b. Max a promis à sa fiancée de trouver/ de lui offrir la caverne d'Ali Baba.

Quelles conclusions tirer de cette analyse sémantique des Exis ? Le tableau est assez contrasté, mais au moins trois résultats sont clairs :

1. les Exis sont partiellement compositionnelles : leur sens n'est pas arbitraire et fait intervenir des relations de sens dont les déclencheurs sont à la fois lexicaux et aspectuels (par exemple, un achèvement entraînant un résultat) ;

2. les Exis exhibent de manière claire l'absence d'autonomie référentielle : lorsqu' on est conduit à l'autonomie référentielle de l'argument interne, l'interprétation est littérale ;

3. 1'interprétation littérale n'est pas toujours disponible, et quand ce n'est pas possible, aucun mécanisme sémantique, par exemple la coercion, ne permet de récupérer une interprétation littérale.

Il en découle que la solution au comportement erratique des Exis n'est pas syntaxique, mais pragmatique. Nous allons voir dans la section suivante comment la théorie pragmatique permet de donner une image nouvelle et différente des Exis.

\section{La pragmatique des expressions idiomatiques}

Les premiers travaux sur le sens non-littéral en pragmatique ont tous fait référence aux expressions idiomatiques, qui constituent, dans la compréhension des processus sémantiques et pragmatiques, un stade ultime du changement de sens, ceci malgré le fait que la vocation des expressions à se figer et de leur signification à se cristalliser n'est en fait pas clarifié, ni du point de vue de la description diachronique, ni du point de vue de la théorie du changement linguistique (comme par exemple la théorie de la grammaticalisation).

Deux explications classiques peuvent être données : la première est celle de Morgan (1978), basée sur la différence entre convention de langue et convention d'usage. La seconde fait intervenir la notion d'implicature, et notamment le passage d'une interprétation pragmatique métaphorique à un processus de conventionalisation (Sperber \& Wilson 1986). 
Pour Morgan, les expressions idiomatiques sont l'expression du passage d'une convention d'usage (dont relèvent les implicatures conversationnelles) à une convention de langue. Le test qu'il donne est équivalent à celui de la non-dissémination de Chomsky, puisqu'il prédit qu'une Exi comme to go to the bathroom (litt. aller à la salle de bain, fig. faire pipi) peut recevoir un ajout comme on the carpet (sur le tapis) sans provoquer de contradiction.

(48) My dog went to the bathroom on the carpet.

a. Litt. Mon chien est allé à la salle de bain sur le tapis.

b. Fig. Mon chien a fait pipi sur le tapis.

Le processus de pragmatisation (qui est ici de type métonymique : le lieu pour l'événement) est totalement spécifique à une langue, ici l'anglais. Un tel processus de conventionalisation n'est manifestement pas encore le cas en français, même si aller à la salle de bain peut se comprendre dans ce type de contexte comme aller aux toilettes et plus spécifiquement comme faire pipi :

(49) a. Excusez-moi, après trois bières, il faut que j'aille à la salle de bain.

b. Fig. Il faut que j'aille faire pipi.

Mais le point important est que ce sens n'est pas conventionnel : il ne peut être détaché du lieu, et l'expression ne peut pas, comme en anglais, être complétée par un ajout locatif :

(50) \# Excusez-moi, après trois bières, il faut que j'aille à la cuisine.

(51) ?? Excusez-mois, après trois bières, il faut que j'aille à la salle de bain contre un arbre.

Si l'on admet cette explication, il faudrait donc que toute Exi reçoive un sens pragmatique par convention d'usage (ce serait donc une implicature conversationnelle), et que le temps (ou la fréquence) transforme cette convention d'usage en convention de langue.

Cette explication est bien sûr spéculative, et repose sur deux assomptions :

1. le sens idiomatique des Exis est le résultat d'un processus pragmatique ;

2. la conventionalisation est le fait du temps et/ou de la fréquence.

Cette analyse est-elle consistante avec ce que l'on sait sur les processus pragmatiques ? ${ }^{(12)}$ Il semble en effet plus que plausible que le sens d'une Exi soit effectivement le résultat d'un processus pragmatique, mais plusieurs schémas pragmatiques peuvent être convoqués. Nous en avons souligné deux, l'un de type coercion aspectuelle, l'autre de type métonymique. Nous en développerons un troisième, car il a fait l'objet de plusieurs analyses pragmatiques consacrées la métaphore.

Concernant la seconde assomption (la conventionalisation est le fait du temps et de la fréquence), elle est malheureusement peu documentée sur les expressions idiomatiques ${ }^{(13)}$. L'interaction entre temps et fréquence n'est d'ailleurs pas obligatoire. On sait que les changements se font dans le temps, mais que ceux-ci peuvent être rapides. Prenons une expression introduite dans Du côté de chez Swann, faire catleyas, pour FAIRE L'AMOUR : le sens figuré est ici associé à un événement

(12) Nous laissons de côté la discussion sur la généralisation diachronique de tels processus de changement de sens. $C f$. Heine (2002), pour lequel la conventionalisation est le dernier stade diachronique de la grammaticalisation.

(13) Sur la fréquence des collocations, voir cependant Seretan (2011). 
particulier, où Swann remet sur le corsage d'Odette la fleur qui était malencontreusement tombée à la suite d'une rencontre mouvementée. Mais dans ce contexte, le sens de faire catleyas, associée directement à des gestes intimes, va de soi et n'a pas besoin d'un grand nombre d'usages ni d'une grande fréquence pour que l'expression, nouvelle, reçoive un sens précis pour ses usagers (et pour le lecteur!).

Mais comment définir plus précisément et rigoureusement les processus pragmatiques de changement de sens conduisant à leur figement?

Dans son article consacré à la métaphore, Searle (1982a) fait une distinction importante entre métaphores créatives et métaphore «banales ou mortes ». Voici ce qu'il en dit :

«Les métaphores mortes sont celles qui ont survécu. C'est l'emploi continuel qui les a tuées ; mais leur emploi continuel est l'indice qu'elles satisfont un besoin sémantique donné » (p. 129).

Dans une autre description (p. 164), il résume ainsi le processus conduisant à une métaphore «éteinte » :

«On néglige le sens primitif de la phrase et la phrase acquiert un nouveau sens littéral identique au précédent sens de l'énonciation métaphorique. Il y a glissement du schéma d'énonciation métaphorique [...] au schéma d'énonciation littéral».

Sans détailler la schématisation qu'en fait Searle, l'idée est que le sens de la métaphore figée est identique à un nouveau sens littéral, exploitant l'ancien sens métaphorique. Ainsi, en prenant l'exemple Sophie est un glaçon, son sens métaphorique donné en (52b) devient son nouveau sens littéral :

(52) a. Sophie est un glaçon.

b. Sophie est une personne insensible que rien n'émeut.

Aucun autre effet de sens n'est attendu dans cette ancienne métaphore, et elle s'accommode d'ailleurs facilement de tout un paradigme associant objet froid, ou produisant du froid, à l'ancien sens métaphorique :

(53) a. Sophie est froide.

b. Sophie est un frigidaire.

c. Sophie est un congélateur.

d. Sophie est un iceberg. etc.

On remarquera que tout ce qui est froid n'est pas un prédicat susceptible de communiquer l'absence d'émotion :

(54) a. Sophie est un glacier.

b. Sophie est une patinoire.

c. Sophie est une champ de neige/ une piste de ski, etc.

Ce qui est original dans 1'analyse de Searle est donc que le figement exploite un ancien processus métaphorique, mais s'assimile à un sens littéral. Si l'on ajoute maintenant la contribution de Searle au sens littéral, celle-ci penche très fortement du côté pragmatique, puisque dans son article, " la notion de sens littéral n'a d'application que relative à un ensemble d'assomptions contextuelles ou d'ar- 
rière-plan » (Searle 1982b, 167). En conséquence, le sens figuré d'une Exi ne serait qu'un nouveau sens littéral.

La seconde stratégie d'explication du sens des Exis fait intervenir la notion d'implicature conversationnelle. Le raisonnement est le suivant : les Exis sont d'anciennes métaphores qui se sont conventionnalisées. Si Searle interprète le processus de conventionalisation comme conduisant à un nouveau sens littéral, les approches pragmatiques en tirent une autre conclusion : le sens d'une Exi passerait d'une implicature conversationnelle faible à une implicature conversationnelle forte (Sperber \& Wilson 1986) ${ }^{(14)}$.

Dans la version classique de la théorie de la pertinence (Sperber \& Wilson 1986), une distinction fondamentale est introduite : entre implicature (conversationnelle) ${ }^{(15)}$ forte et faible. La différence tient non à la détermination des implicatures (leur contenu), mais à la force avec laquelle elles sont entretenues. Une implicature forte relève de la responsabilité du locuteur, alors que les implicatures faibles relèvent de celle du destinataire. Dans le cas des métaphores, les métaphores créatives communiquent un ensemble d'implicatures faibles : celles-ci ne peuvent pas être toutes imputées au locuteur, et elles sont donc de la responsabilité des destinataires. Ces implicatures sont conversationnelles, car elles sont dépendantes du contexte. L'idée est que si une hypothèse contextuelle est changée, la conclusion implicitée sera différente.

Prenons un exemple classique :

(55) La femme est l'avenir de l'homme.

Ce vers d'Aragon communique un grand nombre d'implicatures faibles, données en (56) :

(56) a. La femme fait le bonheur de l'homme.

b. Sans femme, l'humanité n'a pas d'avenir.

c. Sans femme, un homme est perdu.

d. Les femmes survivront aux hommes.

etc.

Cette métaphore, créative, contraste dans ses effets avec les métaphores ordinaires, comme (57):

(57) Ta chambre est une porcherie.

Le sens pragmatique (implicature forte) peut être donné en (58) :

(58) a. Ta chambre est sale et en désordre.

b. Ta chambre ressemble à une porcherie.

c. Tu devrais ranger ta chambre.

(14) Il est intéressant de constater qu'il n'y a pas d'approche pragmatique associant le sens d'une Exi à une implicature conventionnelle. Soit il y a un nouveau sens littéral (Searle), soit l'implicature conversationnelle se généralise, tout en restant annulable (la propriété centrale des implicatures conversationnelles). La notion d'implicature conventionnelle n'a jamais été appliquée au sens d'une expression idiomatique.

(15) La TP ne fait pas la distinction entre implicature conventionnelle et conversationnelle : les implicatures conventionnelles relèvent de ce qui est dit (explicature) et les implicatures correspondent aux implicatures conversationnelles particulières. Les implicatures conversationnelles généralisées relèvent ou de ce qui est dit (explicature par spécification) ou de ce qui est implicité (implicatures scalaires). 
Ces implicatures sont difficilement défaisables, et il semble que le locuteur ne puisse pas ne pas les prendre en charge. Par exemple, les énoncés en (59) sont manifestement contradictoires :

(59) a. ?? Ta chambre est une porcherie, mais elle ne ressemble pas à une porcherie.

b. ?? Ta chambre est une porcherie, mais elle n'est pas sale ni désordonnée.

c. ?? Ta chambre est une porcherie, mais tu ne devrais pas la ranger.

Au vu de ces observations, on peut formuler une hypothèse selon laquelle, s'il y a un rapport entre Exis et métaphores figées, c'est que le sens figuré n'est qu'une implicature forte, qui relève de la responsabilité du locuteur. Nous ne sommes pas loin, avec une terminologie différente, de la première approche pragmatique, celle de Morgan, pour qui les Exis sont des conventions de langue. Mais une convention de langue suppose que le sens de l'expression passe par un processus de conventionalisation. Deux solutions sont alors possibles : ou l'on revient à la solution de Searle, pour qui le sens d'une Exi est un nouveau sens littéral, certes relié à un ancien sens métaphorique ; ou alors on passe par la notion d'implicature conventionnelle. Mais des difficultés empiriques apparaissent immédiatement : les implicatures conventionnelles sont non vériconditionnelles et non annulables. Or ces deux critères ne semblent pas facilement satisfaits par les Exis.

(60) teste le critère de non-vériconditionnalité, et (61) celui de non-annulabilité :

(60) ?? A l'heure qu'il est, Max a dû casser sa pipe.

(61) ?? Max a cassé sa pipe, mais il n'est pas mort.

En (60), le sens implicité conventionnellement MOURIR ne peut pas être dans la portée d'un opérateur épistémique (devoir) : cela tient au fait que l'événement de CASSER SA PIPE est subit et imprévisible, et ne peut pas faire l'objet d'une inférence épistémique, comme cela est le cas en (62):

(62) A l'heure qu'il est, Max a dû arriver chez lui.

D’un autre côté, (61) est un énoncé contradictoire, ce qui nous conduit à admettre que le sens de casser sa pipe est une implicature conventionnelle, par définition non-annulable. (63) confirme cette intuition :

(63) Max a cassé sa pipe, donc il est mort.

D'un autre côté, (63) ressemble d'avantage à un enchaînement sur une implication sémantique (entailment). Dans ce cas, le test (Moeschler 2013) ne repose pas sur l'implication d'une phrase positive, mais d'une phrase négative : si une phrase $\mathrm{P}$ implique une phrase $\mathrm{Q}$, alors non-P implique $\mathrm{Q}$ ou non- $\mathrm{Q}$.

Qu'en est-il de (64) :

(64) a. Max n'a pas cassé sa pipe : il n'est donc pas mort.

b. Max n'a pas cassé sa pipe : il n'est pas mort ou il est mort.

Comme seul (64a) est possible - (64b ne pouvant expliciter le sens de ne pas casser sa pipe), le test de la négation montre que la relation entre casser sa pipe et MOURIR n'est pas une relation d'implication, à savoir une relation sémantique, au sens fort du terme.

D'un autre côté, (61) peut très bien se comprendre de manière différente, métalinguistique. Le locuteur fait référence à l'expression casser sa pipe, mais ce qu'il 
veut dire est autre chose : Max a bien cassé quelque chose, sa pipe, et donc il ne faut pas comprendre qu'il est mort. Mais dans ce cas, si le sens de casser sa pipe est annulable, alors le sens MOURIR n'est plus une implicature conventionnelle : c'est, au sens traditionnel, une implicature conversationnelle.

On se trouve donc en face des trois solutions suivantes :

1. le sens de l'Exi est une convention de langue : mais cette solution doit être rejetée, car elle ne peut être une implicature conventionnelle ;

2. le sens de l'Exi est un nouveau sens littéral : mais dans ce cas, il faut admettre que toutes les métaphores mortes n'ont plus de relation avec ce qui était leur sens littéral initial. Or nous avons montré que tel était bien le cas $(\S 3)$. 3. Le sens de 1'Exi est une implicature conversationnelle forte. Mais si ce processus est explicable pour les anciennes métaphores, il devient plus opaque pour d'autres phénomènes sémantiques comme la coercion aspectuelle ou encore la métonymie.

Nous sommes donc dans une impasse, du point de vue théorique. Mais si nous revenons sur 1'une de nos hypothèses de la section 3, selon laquelle le sens d'une Exi est compositionnel, alors nous pouvons entrevoir une solution pragmatique.

L'une des hypothèses pragmatiques récentes, en pragmatique lexicale tout au moins (Wilson 2006, Wilson \& Carton 2007, Reboul 2007 pour une analyse critique), est de considérer que l'interprétation pragmatique est le résultat d'un ajustement pragmatique conceptuel. La théorie des concepts ad hoc stipule que l'information conceptuelle encodée linguistiquement est modulée pragmatiquement, et donc que le concept inféré pragmatiquement, ou concept ad hoc, est le résultat d'un processus inférentiel. En d'autres termes, le concept inféré pragmatiquement peut être très différent du concept encodé linguistiquement. Par exemple, le concept ad hoc CÉLIBATAIRE* est en (65) un élargissement du concept encodé, alors qu'il en est une spécification en (66) :

(65) Mon mari est un vrai célibataire : je peux le suivre à la trace avec son linge sale du salon à la salle de bain!

(66) Marie est heureuse : elle vient de rencontrer un célibataire.

En (65), CÉLIBATAIRE* est un élargissement extensionnel, ce qui implique que les individus CÉLIBATAIRES* ne satisfont pas certaines implications logiques du concept, comme par exemple l'inférence (67) :

(67) CÉLibataire(x) $\rightarrow$ NON-MARié(x)

En (66), le concept ad hoc a pour extension un sous-ensemble du concept encodé linguistiquement : CÉLIBATAIRE** a pour explicature des propriétés comme JEUNE, NON ENGAGÉ, DISPONIBLE, CANDIDAT AU MARIAGE, etc. Ces prédications sont inférées pragmatiquement, ce sont donc des explicatures de base.

Revenons maintenant aux Exis, et à notre exemple jouet casser sa pipe. Dans la théorie des concepts ad hoc, le concept CASSER SA PIPE a pour concept ad hoc CASSER SA PIPE*. Le sens idiomatique serait donc un cas particulier de 1'ajustement contextuel d'un concept encodé linguistiquement. Simplement, ce concept a pour entrée lexicale une expression complexe, figée. En second lieu, contrairement aux concepts simples ajustés pragmatiquement à chaque contexte, un concept ad hoc complexe reçoit une fois pour toutes, par un processus qui peut être le résultat de parcours diachroniques multiples, un sens idiomatique. 


\section{La motivation du processus pragmatique}

La dernière étape de l'explication pragmatique (lexicale) est de savoir pourquoi un tel processus peut se produire En d'autres termes, quel serait le bénéfice, pour la cognition et la communication, de disposer d'un répertoire, stable mais plastique, d'expressions idiomatiques. Dans le cadre de la théorie de la pertinence, et des concepts ad hoc en particulier, une telle réponse a été fournie.

Les idiomes auraient pour fonction la création de chemins préférentiels (VegaMorano 2001,2005) : plus l'idiome est utilisé, plus le chemin est balisé, connu, et donc plus son sens est accessible. Le fait que les idiomes soient pour la plupart d'anciennes métaphores, dont l'usage, et donc le sens, s'est généralisé, n'est pas crucial ici. Ce qui l'est, c'est l'idée de chemin préférentiel. Cette idée doit être mise en rapport avec la procédure de compréhension de la Pertinence (Wilson \& Sperber 2004, 613):

«a. Suivez le chemin du moindre effort dans le calcul des effets cognitifs : testez les hypothèses interprétatives (désambiguïsation, résolution de la référence, implicatures, etc.) dans l'ordre de leur accessibilité.

b. Arrêtez lorsque vos attentes de pertinence sont satisfaites (ou abandonnées)».

Du point de vue de la pertinence, la fonction d'une expression idiomatique fait sens dans le dispositif pragmatique général d'interprétation des énoncés. L'hypothèse de la Pertinence est en effet celle de la sous-spécification linguistique (ou sémantique) du sens des énoncés. Cela veut dire que l'interprétation est essentiellement le résultat d'un processus d'enrichissement pragmatique (calcul des explicatures) et de calcul des implicatures (prémisses et conclusions implicitées). La fonction des Exis est de ce point de vue évidente : le sens d'une Exi est le résultat de la construction d'un concept ad hoc complexe, dont le déclencheur est une forme linguistique spécifiquement attachée au concept. L'accès au concept inféré pragmatiquement n'est donc pas, dans le cas des Exis, le résultat d'un processus pragmatique complexe. Il obéit au contraire à un processus d'accès direct, analytique : l'entrée lexicale permet un accès direct aux concepts inférés pragmatiquement.

L'hypothèse de Vega-Morano prend sens ici : en proposant un chemin préférentiel entre une forme et un sens, les Exis sont un moyen optimal de satisfaire les attentes de pertinence. Le risque évidemment, et donc l'inconvénient, tient au fait que le chemin doit être connu : s'il ne l'est pas, les attentes de pertinence ne peuvent qu'être abandonnées.

\section{Conclusion}

Dans cet article, nous avons tenté de soulever et de discuter les principales questions adressées par la syntaxe, la sémantique et la pragmatique des expressions idiomatiques. Notre conclusion est que l'origine du sens figuré de ces expressions repose essentiellement sur des processus pragmatiques.

La conclusion à laquelle nous sommes arrivés est double : d'une part le sens d'une expression idiomatique ou figée contribue au sens explicite de l'énoncé (et donc à son explicature de base), plutôt qu'à son sens implicite (ou implicature) ; d'autre part ce processus relève d'un enrichissement pragmatique en quoi consiste la création d'un concept $a d$ hoc, ou concept inféré pragmatiquement. La sta- 
bilité des expressions figées, du point de vue de leur forme et de leur sens, est certainement lié à la relation entre le concept encodé linguistiquement et le concept inféré pragmatiquement : cette relation ne suppose plus un enrichissement libre dépendant du contexte, mais correspond à une fixation pragmatique du sens de l'expression. Il en ressort que si le sens figuré peut trouver une motivation pragmatique, il se trouve être fixé une fois pour toute dans l'entrée lexicale du concept.

La difficulté associée aux expressions idiomatiques n'est ainsi plus théorique, mais pratique : seule 1'exposition à leurs usages permet la fixation du sens inféré pragmatiquement. Il est grand temps que des études expérimentales voient le jour afin de savoir comment le sens pragmatique est attaché à leurs entrées lexicales. On souhaiterait mentionner ici l'étude de Siloni, Horvath, Klunover \& Wexler (2013) qui porte sur les idiomes en hébreu (voir aussi Horvath \& Siloni 2009). Le but de cette étude est de montrer le lien entre l'organisation du lexique mental et la façon dont les expressions idiomatiques sont stockées. Les résultats de leurs expériences montrent que les sujets sont sensibles aux traits grammaticaux des expressions idiomatiques, plus exactement à leurs propriétés morpho-syntaxiques, ce qui signifie qu'elles sont stockées dans le lexique mental et non pas dans un module extra-grammatical. De plus, les expressions idiomatiques sont stockées comme des sous-entrées de leur tête (lexicale) et non pas comme des entrées indépendantes ( $c f$. la discussion en section 2). Ceci est compatible avec l'interprétation pragmatique que nous avons donnée, puisque le stockage des expressions idiomatique relève semble-t-il du niveau de représentation abstraite du lexique mental.

\section{Bibliographie}

BRESNAN, J. (1982), «The passive in lexical theory », in J. Bresnan (ed.), The Mental Representation of Grammatical Relations, Cambridge, Mass., MIT Press.

Chiflet, J.-L. (1985), Sky my husband! Ciel mon mari !, Paris, Hermé.

Chomsky, N. (1980), Rules and Representations, Oxford, Basil Blackwell.

DOETJES, J. \& ROORYCK, R. (2003), « Generalizing over quantitative and qualitative constructions ", in M. Coene \& Y. D'hulst (éds), From NP to DP Volume 1: the syntax and semantics of noun phrases, Amsterdam, John Benjamins, 277-296.

FAUCONNIER, G. (1984), Espaces mentaux, Paris, Minuit.

GAATONE, D. (1981), «Les locutions verbales : pour quoi faire?», Revue Romane 144, 49-73.

- (2000), « À quoi sert la notion d'expression figée ? », in Buvet P.A. et al. (éds), Lexique, Syntaxe et Sémantique. Mélanges offerts à Gaston Gross à l'occasion de son 60e anniversaire, Besançon, Centre Lucien Tesnière, 295-308.

Gazdar, G., E. Klein, G. K. Pullum \& I.A. SAG (1985), Generalized Phrase Structure Grammar, Cambridge, Mass, Harvard University Press and Oxford: Basil Blackwell. 
GRoss, G. (1996), Les expressions figées en français : noms composés et autres locutions, Paris, Ophrys.

HEINE, B. (2002), " On the role of context in grammaticalization », in I. Wischer \& G. Diewald (éds), New Reflections on Grammaticalization, Amsterdam, John Benjamins, 83-101.

HoRVATH, J. \& SiLONI, T. (2009), « Hebrew Idioms: The Organization of the Lexical Component », Brill's Annual of Afroasiatic Languages and Linguistics 1, 283310.

KeEnan, E. (1975), « Some universals of passive in relational grammar », Chicago Linguistic Society Papers 11, 340-352.

Milner, J.C. (1982), Ordres et raisons de langue, Paris, Seuil.

MOESCHLER, J. (1992a), « Idiomes et locutions verbales: à propos de quelques bizarreries syntaxiques et mystères sémantiques ", Terminologie et Traduction 2/3, 135-144.

— (1992b), «Métaphores et idiomes: compositionnalité, interprétabilité et inférence », in Tyvaert J.E. (éd.), Lexique et inférences(s), Paris, Klincksieck, 97114.

— (2013), « Is a speaker-based pragmatics possible? Or how can a hearer infer a speaker's commitment? », Journal of Pragmatics, 43, 84-97.

Moeschler, J. \& A. Reboul (1994), Dictionnaire encyclopédique de pragmatique, Paris, Seuil.

Morgan, J.L. (1978), « Two types of convention in indirect speech acts », in Cole, P. (ed.), Syntax and Semantics 9 : Pragmatics, New York, Academic Press, 261280.

Reboul, A. (2007), Langage et cognition humaine, Grenoble, PUG.

RUWET, N. (1983), «Du bon usage des expressions idiomatiques dans l'argumentation en syntaxe générative ", Revue québécoise de linguistique 13 (1), 9-146.

SEARLE, J.R. (1982a), «La métaphore », in Sens et expression, Paris, Minuit, 121-166. - (1982b), «Le sens littéral», in Sens et expression, Paris, Minuit, 167-188.

Seretan, V. (2011), Syntax-Based Collocation Extraction, Dordrecht, Springer.

Siloni, T., Horvath, J., Klunover, H. \& WeXler, K. (2013), « Idiom storage and the mental lexicon: psycholingusitic reality », Ms., disponible sur le site http://cms.unige.ch/lettres/linguistique/seminaire/media/234/SILONI\%20 ET \%20AL_Idiom\%20Storage\%20psycholinguistic\%20evidence\%20Final \%2013.6.pdf.

SPERBER, D. \& Wilson D. (1986), Relevance. Communication and Cognition, Oxford, Blackwell.

VEGA-MoRENO, R.E. (2001), « Representing and processing idioms », UCL Working Papers in Linguistics 13, 73-107.

- (2005), Creativity and Convention: The Pragmatics of Everyday Figurative Speech, PhD Thesis. University College London, University of London.

Wilson, D. (2006), « Pertinence et pragmatique lexicale », Nouveaux cahiers de linguistique française 27, 33-52.

WILSON, D. \& CARSTON, R. (2007), « A unitary approach to lexical pragmatics: relevance, inference and ad hoc concepts », in Burton-Roberts N. (éd), Pragmatics, London, Palgrave, 230-260.

Wilson, D. \& S PERBER, D. (2004), "Relevance theory », in Horn L.R. \& Ward G. (éds.), The Handbook of pragmatics, Oxford, Blackwell, 607-632. 